\title{
Editorial
}

\section{Review on Artificial Intelligence and Applications in Healthcare}

\author{
Ravish Huchegowda, MBBS, MD ${ }^{\prime *}$; Srinivas Huchegowda, MBBS, MD, DNB, PhD²; Jyothi R. Jain, Mphil, PhD³; \\ Manoj Parthasarathy, MBBS Student ${ }^{4}$; Tharika Shraddha, MBBS ${ }^{5}$; Nagalakshmi C. Sathyanarayanshetty, MBBS, MD; \\ Bharat V. Poojary, BE'; Farhan Zameer, MSc, PhD ${ }^{8}$; Chetan H Gowda, BE, MTech, PhD'; Naveen H. Gowda, BE, MTech'10; \\ Huskur N.Venkatesh, MSc, PhD"'
}

'Department of Neurochemistry, National Institute of Mental Health and Neuro Sciences (NIMHANS), Bangalore 560029, Karnataka, India

${ }^{2}$ Department of Biochemistry, Maulana Azad Medical College, Delhi I I0002, New Delhi, India

${ }^{3}$ Department of Biotechnology, Jain (Deemed-To-Be-University), Jayanagar, $3^{\text {rd }}$ Block, Bangalore 56004I, Karnataka, India

${ }^{4}$ MBBS Student, Mandya Institute of Medical Sciences, Mandya 57 I 40 I, Karnataka, India

${ }^{5}$ General Practitioner, Bangalore 560029, Karnataka, India

${ }^{6}$ Department of Biochemistry, Akash Medical College, Bangalore 560029, Karnataka, India

${ }^{7}$ Software Engineer, Surya Software Systems Private Limited, Bangalore 560029, Karnataka, India

${ }^{8}$ Department of Biochemistry, School of Basic and Applied Sciences, Dayananda Sagar University, Bangalore 560029, Karnataka, India

${ }^{9}$ Department of Electronic and Communication, CMRIT College, Bangalore 560029, Karnataka, India

${ }^{10}$ Department of Electronic and Communication, New Horizon Engineering College, Bangalore 560029, Karnataka, India

"Department of Human Genetics, NIMHANS, Bangalore 560029, Karnataka, India

\section{"Corresponding author}

Ravish Huchegowda, MBBS, MD

Assistant Professor, Department of Neurochemistry, National Institute of Mental Health and Neuro Sciences (NIMHANS), Bangalore 560029, Karnataka, India; Tel. 08026995908, 9741990094; E-mail: docravish@nimhans.ac.in; neuroravish@gmail.com

\section{Article information}

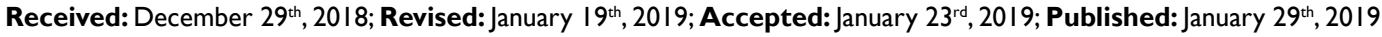

\section{Cite this article}

Huchegowda R, Huchegowda S, Pramer J, et al. Review on artificial intelligence and applications in healthcare. Neuro Open J. 2019;6(I): el-e4. doi: $10.17140 / \mathrm{NOJ}-6-\mathrm{e} 010$

\section{ABSTRACT}

Artificial intelligence (AI) is going to make huge difference in our day to day lives. AI is influencing the way we live and how we interact with the world, and there is much more to come in the years to follow with more advancement. As AI becomes more deeply integrated into our lives, it will become the new infrastructure powering a second industrial revolution. Bridging the link between current nano-sciences and AI, it can boost research in various disciplines and provide a new generation of information and communication technologies that shows a large impact in our society, probably providing the means so that technology and biology merge. Major changes in the education curriculum of medical professionals need to take place. But the rising cost of healthcare may prove to be an independent driving force to develop these technologies; meanwhile health information technology not only improves the quality of care, but also reduces its cost significantly. AI has the potentiality to reduce the cost of healthcare markedly and in future, this may translate into creation of promotional policies to accelerate investment in AI by rewarding the hospitals and the physicians who incorporates it into their workflow. While a terminator-like scenario is unlikely any time soon, the progression of artificial intelligence techniques and its applications will certainly be very exciting.

Keywords

Artificial intelligence; Deep learning; Healthcare; Diagnostics; Imaging.

$\mathrm{M}^{\mathrm{c}}$ edical knowledge has undoubtedly expanded in the epoch of information technology making it impossible for a single human to keep track of all the knowledge. This has led to heavy application of computer and information technology in medicine, which resulted in evolution of artificial intelligence (AI). ${ }^{1}$

The intelligence can be described as the ability to perceive information and retain it as knowledge to be applied towards adaptive behaviors within an environment or context. ${ }^{2}$ These computer systems use a number of different algorithms and decision-making capabilities as well as a vast amount of data, to provide a solution or response to a request. AI is the potentiality of a machine to synthetically imitate intelligence and thought patterns. ${ }^{3}$ It is connected with the simulation of human intelligence processes by machines, especially computer systems. AI involves learning, reasoning and auto-correction. ${ }^{4}$ 
Alan Turing made a turning point in the history of 1950's in which he speculated about the credibility of creating machines with true intelligence. He noted that "intelligence" is onerous to define and formulated famous Turing test. Turning test soon became the first serious approach towards artificial intelligence, test was a method of finding out whether or not a computer is able to think like a real person.

In 1956 John McCarthy was considered as the father of AI, assembled a Dartmouth conference to pull the talent and expertise of others interested in machine intelligence. From that point on, the Dartmouth conference put forth together the founders in $\mathrm{AI}$ and served to lay the preliminary work for the upcoming AI researchers. ${ }^{6}$ Diverse areas of AI include: neural networks, game theory, programming languages, expert systems, genetic algorithms, speech/handwriting recognition, vision, robotics, search algorithms, learning systems, natural language processing, common knowledge databases, logic, agents, planning, prediction and automation software.

\section{COMPONENTS OF ARTIFICIAL INTELLIGENCE INCLUDE}

\author{
- Perception \\ - Learning \\ - Reasoning \\ - Problem-solving \\ - Language-understanding
}

AI has multitudinous targets as mentioned with different techniques used for each. The foremost and much significant are artificial neural networks (ANNs) and an advanced version known as deep learning.

Artificial neural networks are algorithms which were inspired by the biological process of the brain. An ANN is set up for a specific application, such as pattern recognition or data classification. Deep learning, while sounding flashy is a term to describe certain types of neural networks and related algorithms that consume often raw input data.

Artificial neural networks and complex deep learning techniques are some of the most capable AI tools to resolve very complex problems and will continue to spread and leverage in the future. ${ }^{7}$ A person can train their AI with their proclivity for levels of interaction and frequency. Unlikely, AI is not magic, it's applied science. But most importantly, AI deals with probabilities, not absolutes. The field of artificial intelligence has significantly unfolded many things with introduction of a number of sophisticated algorithms, some of which are capable of selflearning. Today Microsoft, Google, Apple, IBM and Amazon have adopted AI and have already succeeded in providing service to consumers. $^{8}$

The Watson platform has been used in a number of disciplines within healthcare which comprises payers, oncology and patient risk assessment. ${ }^{9}$

\section{APPLICATIONS OF ARTIFICIAL INTELLIGENCE}

1. Personal health virtual assistant-Virtual patient assistant in monitoring and helping patients and health care providers. ${ }^{10}$

2. Advanced analytics and research-For example, in oncology cases, AI helps to detect abnormalities in X-rays and MRIs, in genomics to perform complex processing and in precision medicine to provide assistance in creating highly customized treatments for individual patients. ${ }^{11}$

In the example of IBM Watson, the AI has successfully applied its abilities to process structured and unstructured patient data. In the field of oncology, IBM Watson can provide evidencebased treatment recommendations for cancer patients. ${ }^{12}$

3. Personal life coach-Several hospitals have already initiated life coaching services as part of their overall care, however, with today's robust AI capabilities and mobile apps, patients can receive feedback on a number of data elements captured on their phone or wearable devices. As a result, AI provides personal life coach and creates a customized experience for each individual patient and offers proactive alerts as a feedback to physicians. ${ }^{13}$

4. Healthcare bots-Customer service and health care bots are the evolving areas in $\mathrm{AI}$ which is going to gain adoption soon as part of what healthcare providers offer. A Bot is an AI application patients can interact through a chat window on a website or via telephone to receive help with their requests. Bots helping 24/7 is near future emerging concept. ${ }^{14}$

\section{Applications and Expert Systems of Artificial Intelligence Getting in Biomedical Field}

a) Fuzzy expert systems in medicine: Fuzzy logic is a technique which uses data-handling purpose that allows ambiguity particularly used in medical field. This technique is used in various medical fields such as multiple logistic regression analysis and also used for the diagnosis of many diseases like lung cancer, acute leukemia, breast and pancreatic cancer. It can also predict the survival of patients suffering from breast cancer. ${ }^{15}$

b) Artificial intelligence to improve hospital inpatient care: The most welcome method of AI is a clinical decision support system. This expert system initially focuses on diagnosing the condition of the patient by giving demographic information and his symptoms. It recommends antibiotics for the treatment of infected patients. ${ }^{16}$ This method uses Bayesian network which helps in diagnosis of varying form of cancer and for unexpected heart diseases. ${ }^{17}$

c) Artificial intelligence approaches for medical image classification: Some applications of AI are used for diagnostic sciences in categorization of different type of biomedical image such as identifying tumors in brain. Decision-support tools and model-based intelligent system are the most useful methods for the medical image classification for analysis and evaluation purpose. ${ }^{18}$ described disorder would have been categorized as Chronic 
progressive external ophthalmoplegia (CPEO) plus.

\section{Application of ANN in Health Care Domain}

a) MRI brain tumor analysis: To classify images in diagnostic science, ANN techniques are used. Least squares support vector machines (LSSVM) is another mechanism used for the diagnosis of normal and abnormal areas of brain from data of magnetic resonance imaging (MRI). Because of autonomous way to classify MRI image, it shows result with greater accuracy than other classifiers. $^{19}$

b) Gastroenterology: This technique works by merging the methods of fuzzy systems and radial based function. ${ }^{20}$

c) Heart disease classification: Artificial neural network has substantiated its ability by working on the classification of heart disease. ${ }^{21}$ In this technique for the classification of stroke, the input of sensor is given to the system that uses forward feed network with the rule of back propagation way.

d) Decision support system to diagnose nodules: Through the concept of ANN, the new proposed system is decision support system (DSS). A decision support system diagnoses nodules into benign and malignant or identifies its severity by analyzing the collected data. ${ }^{6}$

Regardless of its ability to see and listen, AI also smells. Humans aren't particularly aware of the richness of information that can be transmitted through the air and can be perceived by a highly sensitive olfactory system. ${ }^{22}$ AI brought change into that by introducing machines in the laboratory which detects very small amounts of substances in the air. Those machines are called gaschromatography mass-spectrometers or gas chromatographymass spectrometry (GC-MS), which analyses the air to discover thousands of different molecules known as volatile organic compounds. AI system helps to reveal the illness by smelling human breathe substances. ${ }^{23}$

\section{CONCLUSION}

Artificial intelligence is going to make huge difference in our day to day lives. AI is influencing the way we live and how we interact with the world, and there is much more to come in the years to follow with more advancement.

Bridging the link between current nano-sciences and $\mathrm{AI}$, it can boost research in various disciplines and provide a new generation of information and communication technologies that shows a huge scope. Major changes in the education curriculum of medical professionals need to take place. But the rising cost of healthcare may prove to be an independent driving force to develop these technologies; meanwhile, health information technology not only improves the quality of care but also reduces its cost significantly. AI has the potentiality to reduce the cost of healthcare markedly and in future, this may translate into creation of promotional policies to accelerate investment in AI by rewarding healthcare setup and healthcare provider incentives for using AI applications.

While a terminator-like scenario is unlikely any time soon, the progression of artificial intelligence techniques and its applications will certainly be very exciting.

\section{CONFLICTS OF INTEREST}

The authors report no conflicts of interest.

\section{REFERENCES}

1. Modi S. Artificial intelligence and neurology. J Biomed Syst Emerg Technol. 2016; 3:

2. Katamreddy S, Riordan D, Doody P. Artificial Calf Weaning Strategies and the Role of Machine Learning: A Review. Papaer presented at: Irish Signals and Systems Conference (ISSC). 20-21 June 2017; Killarney, Ireland. doi: 10.1109/ISSC.2017.7983634

3. Zackova E. Intelligence explosion quest for humankind. Beyond Artificial Intelligence. 2015; 9: 31-43. doi: 10.1007/978-3-319-096681_3

4. Dash D. Autonomy and Artificial Intelligence: The Future Ingredient of Area Denial Strategy in Land Warfare. New Delhi, India: KW Publishers Pvt Ltd. 2018.

5. Dembski W, Kushiner J, (eds). Signs of Intelligence: Understanding Intelligent Design. Ada, Michigan, USA: Brazos Press. 2001.

6. Kallio J. Support Vector machine and deep learning in medical applications. University of Tampere. 2017. Web site. http:/ / tampub. uta.fi/bitstream/handle/10024/101911/GRADU-1504009242. pdf? sequence $=1 \&$ is Allowed $=\mathrm{y}$

7. Caronongan A, Gorgui-Naguib H, Naguib RNG. The Development of Intelligent Patient-Centric Systems for Health Care. New York, USA: Springer International Publishing. 2018.

8. Gubbi J, Buyya R, Marusic S, Palaniswami M. Internet of things (IoT): A vision, architectural elements, and future directions. Future Generation Computer Systems. 2013; 29: 1645-1660. doi: 10.1016/j. future.2013.01.010

9. Salman M, Wahab Ahmed A, Ahmad Khan O, et al. Artificial intelligence in bio-medical domain: An overview of AI based innovations in medical. International Journal of Advanced Computer Science and Applications. 2017; 8: 319-327.

10. Turban E, Leidner D, McLean E, Wetherbe J. Information Technology for Management, $6^{\text {th }}$ ed. New Jersey, USA: John Wiley \& Sons; 2008.

11. Torkamani A, Andersen KG, Steinhubl SR, Topol EJ. Highdefinition medicine. Cell. 2017; 170: 828-843. doi: 10.1016/j. 
cell.2017.08.007

12. Mitra S, Shankar BU. Integrating radio imaging with gene expressions toward a personalized management of cancer. IEEE Transactions on Human-Machine Systems. 2014; 44: 664-677. doi: 10.1109/THMS.2014.2325744

13. Gustafson DH, McTavish FM, Chih MY, et al. A smartphone application to support recovery from alcoholism: A randomized clinical trial. JAMA psychiatry. 2014; 71: 566-572. doi: 10.1001/ jamapsychiatry.2013.4642

14. Diana F. Artificial Intelligence Intersects with Nanotechnology. Web site: https://medium.com/@frankdiana/artificialintelligence-intersects-with-nanotechnology-a674204daa31. 2016.

15. Jain LC, Kandel A, Teodorescu HN. Furay and Neuro-Furay Systems in Medicine. Florida, USA: CRC Press. 2017.

16. Neill DB. Using artificial intelligence to improve hospital inpatient care. IEEE Intelligent Systems. 2013; 1: 92-95. doi: 10.1109/ MIS.2013.51

17. Krishnaiah V, Narsimha DG, Chandra DN. Diagnosis of lung cancer prediction system using data mining classification techniques. International Journal of Computer Science and Information
Technologies. 2013; 4: 39-45.

18. Müller H, Michoux N, Bandon D, Geissbuhler A. A review of content-based image retrieval systems in medical applicationsclinical benefits and future directions. Int J Med Inform. 2004; 73: 1-23. doi: 10.1016/j.ijmedinf.2003.11.024

19. Vas P. Artificial-Intelligence-Based Electrical Machines and Drives: Application of Furzy, Neural, Furzy-Neural, and Genetic-AlgorithmBased Techniques. England, UK: Oxford University Press. 1999.

20. Jang JS. ANFIS: adaptive-network-based fuzzy inference system. IEEE Transactions on Systems, Man, and Cybernetics. 1993; 23: 665-685. doi: 10.1109/21.256541

21. Hudson DL, Cohen ME. Use of Intelligent Agents in the Diagnosis of Cardiac Disorders. Paper presented at: Computers in Cardiology. 22-25 September 2002; Memphis, TN, USA. 633-636. doi: 10.1109/CIC.2002.1166852

22. Beaver BV. Feline Behavior-E-Book. Philadelphia, USA: Saunders Press: 2003.

23. Turner AP, Magan N. Electronic noses and disease diagnostics. Nat Rev Microbiol. 2004; 2: 161-166. doi: 10.1038/nrmicro823 